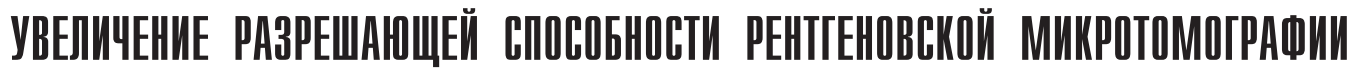

\author{
А. А. Штырляева ${ }^{1}$, А. В. Журавлев ${ }^{2}$ \\ ${ }^{1}$ Национальный минерально-сырьевой университет «Горный», Санкт-Петербург; Shtirlyaeva_aa@mail.ru \\ ${ }^{2}$ Институт геологии Коми НЦ УрО РАН, Сыктывкар; micropalaeontology@gmail.com \\ Предлагается способ увеличения разрешающей способности по рентгеновской плотности рентгеновских микро- \\ томографов за счет фильтрации рентгеновского излучения фильтрами с узкой полосой пропускания. В качестве опти- \\ мального фильтра для томографов SkyScan при использовании ускоряющего напряжения 100-130 кВ предлагается \\ использовать свинецсодержащее стекло толщиной 2.14 мм.
}

Ключевые слова: микротомография, разрешение, рентгеновская плотность, ускоряющее напряжение, фильтр, керн.

\section{IMPROVIIG X-RAY MICROTOMOGRAPHY RESOLUTION}

\author{
A. A. Shtyrlyaeva ${ }^{1}$, A. V. Zhuravlev ${ }^{2}$ \\ ${ }^{1}$ Mining University, Saint-Petersburg \\ ${ }^{2}$ Institute of Geology of Komi SC UB RAS
}

We suggest the method to increase the radiodensity resolution of the industrial microtomographes (SkyScan). The method is based on the filter with a narrow transmission spectrum (60-90 keV with 100-130 kV source voltage). The filter is composed of lead-containing glass $2.14 \mathrm{~mm}$ thick.

Keywords: X-ray microtomography, spatial resolution, radiodensity, source voltage, filter, core sample.

\section{Введение}

Одним из основных недостатков рентгеновских микротомографов в сравнении с томографами, работающими на синхротронном излучении, является относительно низкое разрешение по рентгеновской плотности. Это обусловлено полихромностью рентгеновского излучения трубки, служащей источником излучения. Широкий спектральный диапазон используемого излучения приводит к тому, что наблюдаемые на томограммах рентгеновские плотности являются интегральными. Это сильно затрудняет разделение минеральных фаз, делает их границы размытыми. Кроме того, такая ситуация приводит к невозможности аналитического (по расчетной рентгеновской плотности) разделения на томограммах даже известных минеральных фаз. Как правило, задача разделения на томограмме минеральных фаз решается эмпирически путем сравнения с образцами известного состава. Но и такое решение не дает абсолютно надежного результата, ибо на расчетную рентгеновскую плотность на томограммах оказывают влияние форма и размер (геометрия) исследуемого образца, а также его пространственные соотношения с объектами другой плотности. Например, наблюдается кажущееся снижение рентгеновской плотности у объектов, заключенных в рентгеноплотную матрицу, при этом снижение тем существенней, чем выше рентгеновская плотность матрицы.

Для однородных объектов этот эффект в ряде случаев может проявиться в появлении кажущейся рентгеноплотной каемки на границе раздела образец/воздух (beam hardering). В рентгеновских томографах указанные недостатки частично компенсируются использованием фильтров (обычно Al, $\mathrm{Cu}$, бронза), которые отсекают низкоэнергетическую часть спектра, и применением математической обработки при реконструкции томограммм (компенсация “увеличения жесткости” излучения).

Недостатком алюминиевых и медных фильтров является отсечение только длинноволновой части спектра.
Оставшаяся после фильтрации часть спектра, как правило, характеризуется неравномерно распределенной интенсивностью и существенной шириной (более 70 кэВ) (рис. 1).

Преодоление указанных недостатков возможно, если использовать фильтр-монохроматор с относительно узкой полосой пропускания рентгеновского излучения. В этом случае излучение будет ближе к монохромному, а кажущиеся рентгеновские плотности и их соотношения на томограммах ближе к истинным. Можно предположить, что такими свойствами обладает фильтр из свинецсодержащего стекла благодаря характерному распределению коэффициента поглощения свинца с пиком в области 90 кэВ и «окном» в диапазоне 60-90 кэВ.

Задача данной работы состоит в экспериментальном сравнении эффективности стандартных фильтров и фильтра на основе свинецсодержащего стекла, обеспечивающего более узкий спектральный диапазон рабочего излучения.

\section{Метод исследования, условия эксперимента и обработка данных}

Метод рентгеновской томографии для исследования реализован на основе томографа SkyScan 1173 (Bruker), оснащенного микрофокусным источником рентгеновского излучения Hamamatsu 130/300 и позволяющего получать томограммы с пространственным разрешением до 6.5 мкм [5]. Съемка проводилась при ускоряющем напряжении 115 кВ, токе 69 мкА, угловом шаге сканирования $0.2^{\circ}$ и пространственном разрешении 16 мкм. Реконструкция томограмм осуществлялась программным обеспечением NRecon (Bruker) с последующей обработкой в программных пакетах DataViewer (Bruker) и VolView 3 (Kitware Inc.).

Проведено экспериментальное исследование возможности повышения разрешения по рентгеновской плотности при использовании в качестве фильтра сви- 


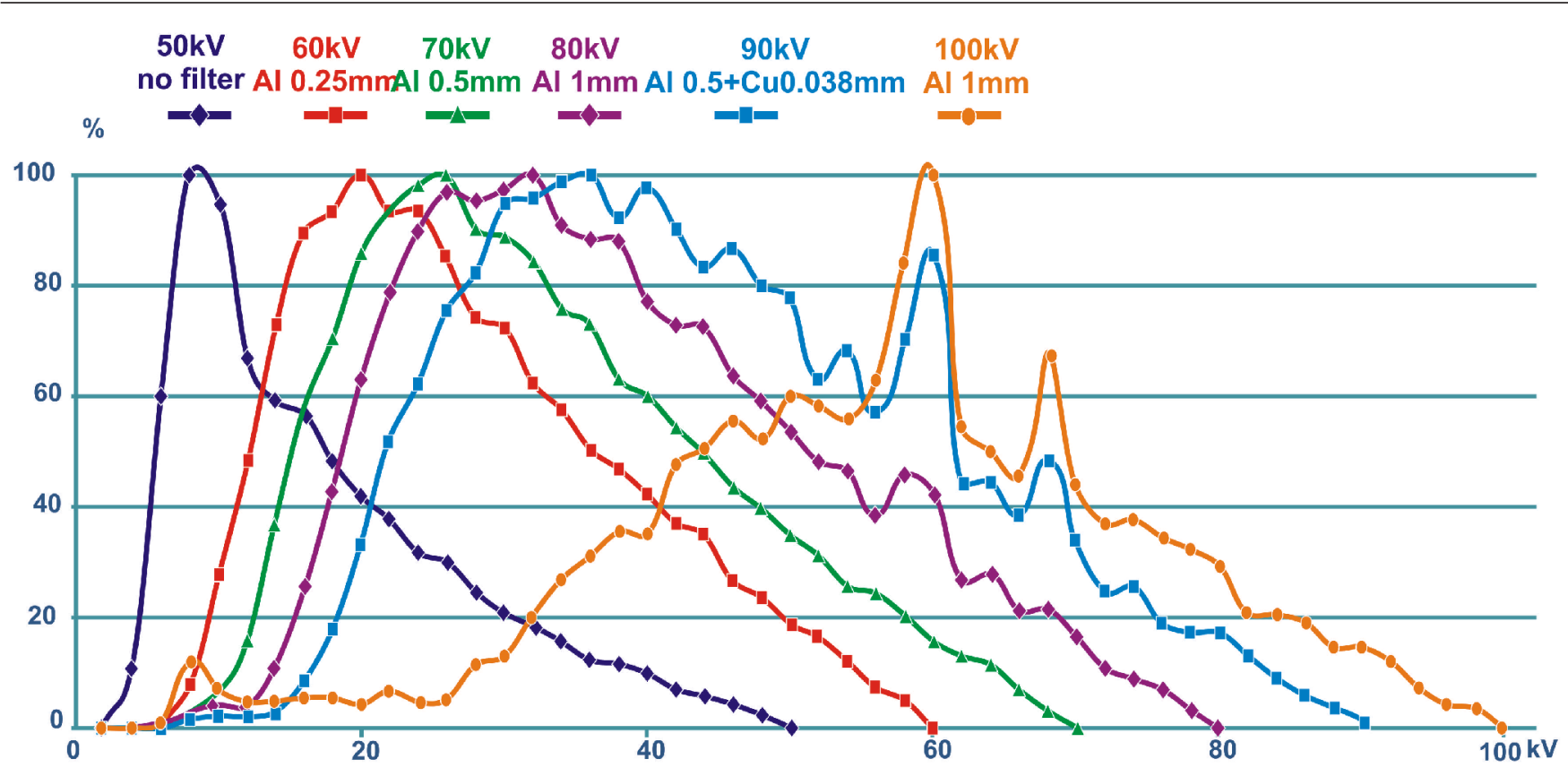

Рис. 1. Спектры рентгеновского излучения в зависимости от заданного ускоряющего напряжения и фильтров для томографов SkyScan [5].

Fig. 1. X-ray spectra as a function of given accelerating voltage and used filters for SkyScan scanners [5].

нецсодержащего стекла толщиной 2.14 мм, обладающего избирательным поглощением рентгеновского излучения в рабочем диапазоне микротомографа SkyScan 1173 (0-130 кэВ). При ускоряющем напряжении около 100 кВ расчетный диапазон фильтрованного излучения - от 60 до 90 кэВ с близким к нормальному распределением интенсивности (рис. 2). Предлагаемая методика съемки с фильтром из свинецсодержащего стекла была опробована на известных образцах кальцита и кварца, принятых за образцы сравнения, а также на образце керна (мелко- зернистый песчаник с карбонатным цементом). Для сравнения те же образцы были сняты с латунным (0.25 мм) и алюминиевым (1 мм) фильтрами.

Оценка эффективности фильтров проводилась путем анализа гистограмм рентгеновской плотности образцов сравнения - кристаллического кварца и биогенного кальцита (рис. 3), а также анализа соответствия измеренных рентгеновских плотностей значениям, расчитанным на основе поглощения монохромного излучения $[3,4]$.

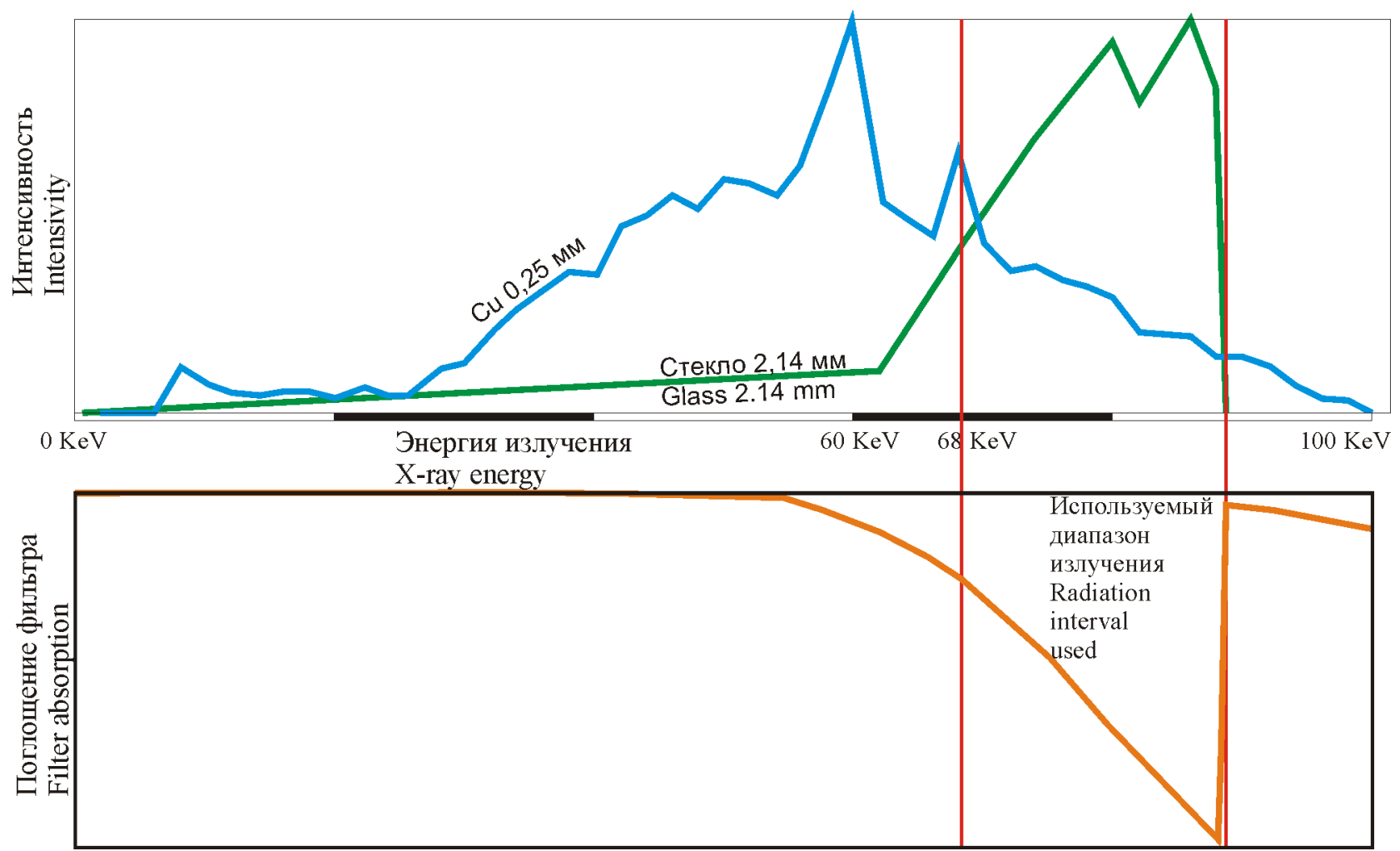

Рис. 2. Сравнение теоретически рассчитанных спектров рентгеновского излучения при использовании медного (0.25 мм) и $\mathrm{Pb}$-стеклянного (2.14 мм) фильтров

Fig. 2.Comparison of theoretically calculated $\mathrm{X}$-ray spectra using $\mathrm{Cu}-0.25 \mathrm{~mm}$ and Glass-( $\mathrm{Pb})-2.14 \mathrm{~mm}$ filters 

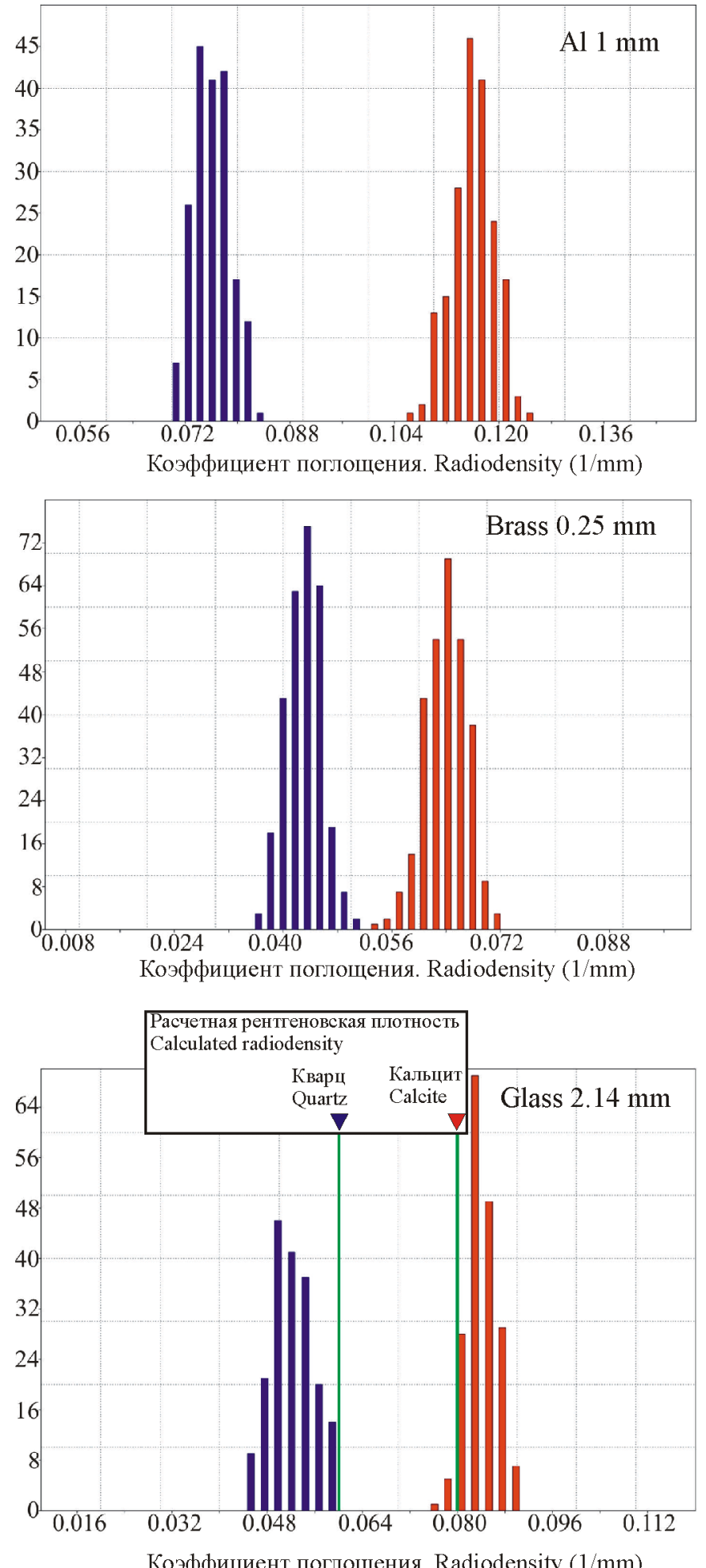

Рис. 3. Гистограммы распределения рентгеновских плотностей в кварце (синий) и кальците (красный) при использовании разных фильтров. Ускоряющее напряжение 115 кB (SkyScan 1173)

Fig. 3. Radiodensity histograms for quartz (blue) and calcite (red) using different filters. Source voltage $115 \mathrm{kV}$ (SkyScan 1173).

\section{Обсуждение результатов}

Использование стандартного алюминиевого фильтра (1 мм) обеспечивает хорошее разделение по рентгеновской плотности кварца и кальцита, однако диапазоны вариаций рентгеновских плотностей этих минералов получаются довольно широкими (0.014 1/мм и 0.019 1/мм соответственно) (рис. 3). При съемке с латунным фильтром (0.25 мм) диапазоны вариаций становятся больше
(0.016 1/мм и 0.020 1/мм соответственно), а различия по рентгеновской плотности - ниже. Более того, в этом случае распределения рентгеновской плотности кальцита и кварца практически смыкаются (рис. 3), делая эти минералы плохо различимыми на томограмме. Использование стеклянного фильтра дает результаты с хорошим разделением кальцита и кварца, при этом диапазоны вариаций рентгеновских плотностей этих минералов уже, чем при съемке с алюминиевым или латунным фильтром (0.014 1/мм как для кальцита, так и для кварца). Более того, полученные рентгеновские плотности имеют близкие значения с полученными для монохромного излучения при энергии около 70 кэВ [3, 4] (рис. 3).

Таким образом, результаты эксперимента (съемки) показали, что использование фильтра из свинецсодержащего стекла (2.14 мм) обеспечивает низкую дисперсию рентгеновских плотностей образцов сравнения в сочетании с хорошим разделением кальцита и кварца по рентгеновским плотностям (рис. 3). По значениям рентгеновских плотностей кальцита и кварца результат съемки с использованием $\mathrm{Pb}$-стеклянного фильтра (ускоряющее напряжение 115 кВ) ближе всего к расчетным значениям для энергии 70-75 кэВ. Применение других фильтров, обладающих широкой полосой пропускания, не позволяет сопоставить получаемые на томограммах рентгеновские плотности с какой-либо определенной энергией из-за существенной полихромности излучения.

Близкое сходство расчетных и измеренных рентгеновских плотностей позволяет использовать при интерпретации томограмм таблицы плотностей минеральных фаз (например, MuCalcTool и база данных NIST XCOM) [3, 4] с возможностью сопоставления выделенных фаз с конкретными минералами при наличии дополнительных данных о предполагаемом составе.

Примером подобной диагностики может служить интерпретация одного из сечений томограммы терригенного керна (Северо-Комсомольское месторождение, Ямало-Ненецкий автономный округ) (рис. 4). Образец керна представлен тонкозернистым аркозовым песчаником с карбонатным цементом. По данным оптической микроскопии, состав обломочных зерен кварц, полевой шпат, состав цемента - кальцит, сидерит, присутствует тонкая вкрапленность аутигенных сульфидов. Экспериментальная РТ-съемка керна происходила с одновременным размещением в рабочем поле томографа образцов сравнения - кристаллов кальцита и кварца. Результаты разделения на фазы сегментации томограммы - по рентгеновским плотностям кварца, кальцита, сидерита и пирита демонстрируют хорошую сходимость с данными, полученными по данным оптической микроскопии при исследовании прозрачных шлифов, что указывает на правильность выполненной интерпретации фаз (рис. 4).

\section{Заключение}

Таким образом, использование на микротомографе SkyScan фильтра из свинецсодержащего стекла толщиной 2.14 мм с ускоряющим напряжением 115 кВ позволяет получить рабочее рентгеновское излучение с энергией около 70-75 кэВ. Относительно узкий спектральный диапазон получаемого излучения повышает достоверность сегментации позволяет более надежно разделять на томограммах минеральные фазы по рентгеновской плотности. 


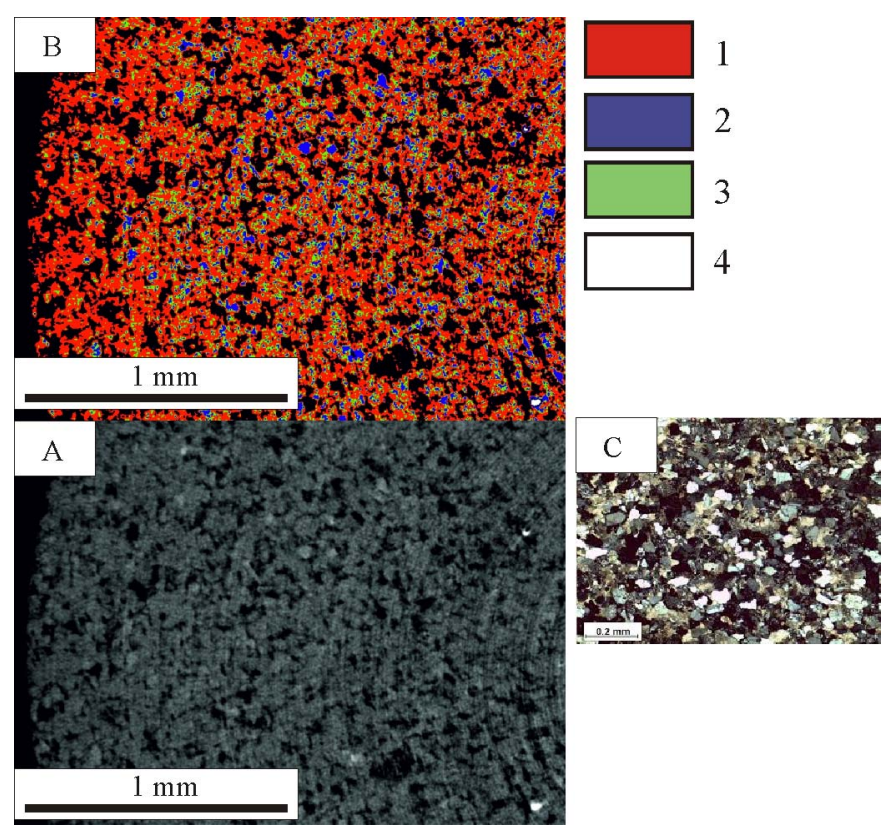

Рис. 4. Сечение томограммы образца керна (аркозовый песчаник) (SkyScan 1173, 115 кB, фильтр Pb-стекло): А - исходная томограмма; В - результаты сегментации по рентгеновским плотностям с интерпретацией минерального состава (1 - кварц, 2 - кальцит, 3 - сидерит, 4 - пирит); С-микрофотография прозрачного шлифа

Fig. 4. Virtual section of tomogram of core sample (arkose sandstone) (SkyScan 1173, $115 \mathrm{kV}$, glass-(Pb) filter). A - raw tomogram; B result of the radiodensity-based segmentation with interpretation of mineral composition ( 1 - quartz, 2 - calcite, 3 - siderite, 4 - pyrite); $\mathrm{C}-$ microphoto of thin-section

\section{Литература • References}

1. Arns C. H., Bauget F., Limaye A., Sakellariou A., Senden T. J., Sheppard A. P., Sok R. M., Pinczewski W. V., Bakke S., Berge L. I., Oren P.-E., and Knackstedt M. A. Pore-Scale Characterization of Carbonates Using X-Ray Microtomography // Society of Petroleum Engineers Journal. Volume 10, Number 4. 2005, pp. 475-484.

2. Kaczmarczyk J., Dohnalik M., Zalewska J. Evaluation of Carbonate Rock Permeability, with the Use of X-ray Computed Microtomography // NAFTA-GAZ, LXVII. N 4. 2011. pp. 233-239.

3. MuCalcTool. http://www.ctlab.geo.utexas.edu/ software/mucalctool/

4. NIST XCOM database at http://www.nist.gov/pml/data/ ffast/index.cfm

5. http://bruker-microct.com/home.htm 